Luke E. Grzeskowiak, Sze Wen Lim, Alicia E. Thomas, Usha Ritchie and Andrea L. Gordon Audit of domperidone use as a galactogogue at an Australian tertiary teaching hospital

Journal of Human Lactation, 2013; 29(1):32-37

(C) The Author(s) 2013

Reprinted by permission of SAGE Publications.

Published version available via DOI: http://dx.doi.org/10.1177/0890334412459804

\title{
PERMISSIONS
}

https://au.sagepub.com/en-gb/oce/journal-author-archiving-policies-and-re-use

Most SAGE journals are published under SAGE's Green Open Access policy, which allows you, as author, to re-use your Contribution as indicated below. For a list of titles that are exceptions to this policy, please scroll down to the bottom of the page.

Green Open Access policy:

Version 2 original submission to the journal with your revisions after peer review, often the version accepted by the editor (author accepted manuscript)

Version 3 copy-edited and typeset proofs and the final published version

- Once the Contribution has been accepted for publication, you may post the accepted version (version $\mathbf{2}$ ) of the Contribution on your own personal website, your department's website or the repository of your institution without any restrictions.

- You may not post the accepted version (version 2) of the Contribution in any repository other than those listed above (i.e. you may not deposit in the repository of another institution or a subject repository) until 12 months after first publication of the Contribution in the journal.

When posting or reusing your Contribution under this policy, appropriate credit must be given to the SAGE journal where the Contribution has been published, as the original source of the content, as follows: Author(s), Article Title, Journal Title (Journal Volume Number and Issue Number) pp. xx-xx. Copyright (c) [year] (Copyright Holder). Reprinted by permission of SAGE Publications. Additionally, please provide a link to the appropriate DOI for the published version of the Contribution on the SAGE Journals website (http://journals.sagepub.com).

\section{March 2018}

http://hdl.handle.net/2440/94368 
Audit of Domperidone Use as a Galactagogue at an Australian Tertiary Teaching Hospital

Luke E Grzeskowiak, Sze Wen Lim, Alicia Thomas, Usha Ritchie, Andrea L Gordon

Luke E Grzeskowiak, BPharm(Hons), GCertClinEpi, Quality Use of Medicines and Pharmacy Research Centre, School of Pharmacy and Medical Sciences, Sansom Institute for Health Research, University of South Australia, Adelaide, South Australia, Australia

Sze Wen Lim, BPharm(Hons), School of Pharmacy and Medical Sciences, University of South Australia, Adelaide, South Australia, Australia

Alicia E Thomas, BPharm, Pharmacy Department, Women's and Children's Hospital, Adelaide, South Australia, Australia

Usha Ritchie, BPharm(Hons), Pharmacy Department, Women's and Children's Hospital, Adelaide, South Australia, Australia

Andrea L Gordon, PhD (Medicine), School of Nursing and Midwifery, Sansom Institute for Health Research, University of South Australia, Adelaide, South Australia, Australia. 


\section{Corresponding Author:}

Luke Grzeskowiak, Quality Use of Medicines and Pharmacy Research Centre, School of Pharmacy and Medical Sciences, Sansom Institute for Health Research, University of South Australia, GPO Box 2471, Adelaide SA 5001, Australia.

Phone: +61 883022403

Fax: +61 883021209

E-mail: grzly001@mymail.unisa.edu.au

Abstract Word Count: 249

Manuscript Word Count: 3,328

\section{Well Established:}

Insufficient milk supply is one of the most commonly reported reasons for discontinuation of infant breastfeeding. While evidence supports the efficacy of domperidone in initiating and maintaining milk supply, knowledge of how domperidone is actually used in clinical practice is scarce.

\section{Newly Expressed:}

The number of women dispensed domperidone increased significantly from $<0.5 \%$ in 2000 to $>5 \%$ in 2010 . Following a comparison to existing evidence, current practice appears to be largely based on anecdotal evidence, highlighting the need for welldesigned randomised controlled trials. 


\section{Abstract}

Background: Domperidone is often used to promote lactation among women who have difficulty breastfeeding.

Objective: To examine prescribing and dispensing practices of domperidone at the Women's and Children's Hospital (WCH), Adelaide.

Methods: A retrospective audit of domperidone dispensing among women with singleton pregnancies who delivered at the WCH between January 2000 and July 2010 was undertaken. Women dispensed domperidone were identified using WCH Pharmacy Dispensing Records. Maternal and infant clinical data was obtained from the WCH Perinatal Statistics Collection. A case note audit for a random sample of 261 of these women and their infants was undertaken to collect prescribing and additional clinical data.

Results: From 2000 to 2010, 1,605 women were dispensed domperidone. There was a steady increase in the percentage of women dispensed domperidone, from $<0.5 \%$ in 2000 to $>5 \%$ of total $\mathrm{WCH}$ pregnancies in 2010 . Over time, the percentage of women who received $>1$ domperidone dispensing remained consistent (20\%), as did the median number of days (12) from delivery to first domperidone dispensing. Multiparous women were more likely to receive domperidone within 3 days following delivery compared to primiparous women ( $8 \%$ vs. $4 \%$ ). Most women ( $80 \%)$ received directions to take domperidone according to a standard tapering dosing regimen over 12 days. Surprisingly, $60 \%$ of women had no documentation of being assessed by a lactation consultant. 
Conclusion: From 2000 to 2010 there was a significant increase in domperidone dispensing. With a lack of clinical evidence to guide use, current practice appears to be based on anecdotal evidence. 


\section{Background}

Exclusive breastfeeding for the first six months of life is recognised as the optimal form of nutrition to support the growth and development of term and preterm infants. ${ }^{1-3}$ Breastfeeding has numerous short and long-term benefits. These include the psychological benefits of maternal-infant bonding, ${ }^{2,4}$ and benefits to the infant including the provision of passive and long-lasting active immunity to infections, ${ }^{1,3,5}$ potential long-term improvements in intellectual development, ${ }^{2,3}$ and reduced future risk of chronic medical conditions such as obesity and diabetes. ${ }^{6}$

Despite the ability of the majority of mothers to be able to produce sufficient quantities of milk for their baby, given the correct support and information, insufficient milk supply, whether real or just perceived, is one of the most commonly reported reasons for discontinuation of infant breastfeeding. ${ }^{7}$ Mothers who are particularly vulnerable to difficulties breastfeeding, are those who deliver infants who are unwell and require supportive care following delivery, such as preterm infants. ${ }^{5,8}$ In many cases these mothers are not able to breastfeed directly which, in addition to the stressful situation of having an unwell infant, can have a detrimental effect on milk supply. However, it is not only women of hospitalised infants who experience difficulty with lactation. Additional risk factors have been identified for difficulty initiating and/or maintaining milk supply including maternal illness, caesarean delivery, maternal smoking, previous breast surgery, mother-infant separation and indirect lactation (i.e. breast pump or manual milk expression). ${ }^{8}$ Furthermore, anxiety, fatigue and emotional stress in mothers can also contribute to poor lactation. ${ }^{9}$

Perceived milk insufficiency can occur as a result of normal postpartum physical breast changes, such as the softening of the breasts around 10 to 14 days postpartum. ${ }^{8}$ This 


\section{Author's Post-Print Version; Copyright - J Hum Lact; doi: 10.1177/0890334412459804}

perception can also be influenced by a lack of confidence in the mother, especially if they have not previously breastfed, lack of support from family and friends and having a limited understanding of lactation physiology. ${ }^{8}$ Additionally, infant symptoms such as irritability, crying and frequent feeding may all be misinterpreted as a lack of breast milk quality or quantity. ${ }^{8}$ In situations of perceived milk insufficiency, non-pharmacological strategies, such as education in correct infant positioning and attachment at the breast, increasing frequency of breastfeeds and mechanical expression are usually sufficient to increase a woman's milk supply. ${ }^{1,8,10,11}$

However, in some situations the use of non-pharmacological strategies are insufficient and some women still struggle to produce or maintain adequate milk supply. This necessitates the need for pharmacological intervention with medications used to augment lactation, known as galactagogues. The most widely studied and commonly used galactagogues include dopamine receptor antagonists, metoclopramide and domperidone, which have both been demonstrated to be effective treatments for initiation and maintenance of lactation. ${ }^{2,5}, 9,10,12-14$

Both of these medications elicit their actions by blocking dopamine $D_{2}$ receptors in the anterior pituitary to stimulate prolactin release. A key difference between agents is that domperidone exerts its pharmacological effects in the peripheral nervous system (PNS), while metoclopramide acts centrally. ${ }^{15}$ While metoclopramide has been used extensively in the past, domperidone is preferred as it does not cross the blood-brain barrier, is associated with less central nervous system (CNS) side effects (i.e. fatigue, irritability, depression), and is secreted in only small amounts into breast milk, compared with metoclopramide. ${ }^{8,9,11}$ 


\section{Author's Post-Print Version; Copyright - J Hum Lact; doi: 10.1177/0890334412459804}

In recent years it has become more common to recommend that mothers with insufficient milk supply (whether real or just perceived) be prescribed dompridone to increase milk production and supply. In recognition of this, the Drug and Therapeutics Committee at the Women's and Children's Hospital (WCH) approved the use of a standardised 'Domperidone Prescription Sheet for Increasing Milk Supply'. This guideline was aimed at standardising domperidone prescribing to a fixed dose-tapering schedule over 12 days. This schedule consisted of one tablet three times a day for five days, followed by one tablet twice a day for three days and then one tablet once a day for four days. Despite its approval and use since 2005, there are concerns that the standardised prescription regimen is largely based on anecdotal evidence rather than evidence from randomized controlled trials. Furthermore, there are concerns that the introduction of this regimen has led to an increase in the prescribing of domperidone prior to adequate investigation of the effects on nonpharmacological interventions to increase milk supply and variability in the time to treatment initiation. Hence, the use of domperidone in clinical practice is of interest. Therefore, the aim of this study was to undertake a retrospective audit of the use of domperidone as a galactagogue at a major tertiary teaching hospital over a 10-year period.

\section{Method}

This was a retrospective audit of women dispensed domperidone who delivered at the WCH in South Australia, Australia, between January 2000 and July 2010. The WCH is a specialist metropolitan tertiary level teaching hospital and South Australia's largest maternity and obstetric service provider, caring for over 4,000 pregnancies each year. The study utilised 
Author's Post-Print Version; Copyright - J Hum Lact; doi: 10.1177/0890334412459804

linkable health administrative data within the $\mathrm{WCH}$, which included the WCH Perinatal Statistics Collection and the WCH Hospital Pharmacy Dispensing Records.

Data relating to women dispensed domperidone were obtained from the WCH Pharmacy Dispensing Records, which contains data on all medications dispensed at the hospital from January 2000. Data available from the dispensing records include dispensing date, type of dispensing (i.e. inpatient, outpatient), quantity supplied and dispensing directions. The electronic dispensing records were then matched to all births recorded in the WCH Perinatal Statistics Collection, using each individual's unique hospital identifier number.

Women were included in the audit if they delivered a singleton infant and received a dispensing for domperidone within 6 months of the date of delivery. This enabled an overall assessment and evaluation of the prescribing and dispensing trends for domperidone over the 10-year period. In addition to the overall assessment, a case note audit was undertaken to obtain prescribing directions and more in-depth clinical data, available only in paper-based medical records, for a random sample of mothers and their corresponding infants. To obtain the random sample, women dispensed domperidone were first stratified according to year of delivery. Where the number of women dispensed domperidone in a particular year was less than 30 , all women for that year were included in the case note audit. Where the number of women dispensed domperidone was greater than 30, a computer-generated random sample was selected. This resulted in a random selection of 261 corresponding mother and infant case notes. Data collected from case notes included dose and duration of domperidone treatment, whether mothers saw a lactation consultant and/or were admitted to the breastfeeding unit and whether they directly requested domperidone. 


\section{Author's Post-Print Version; Copyright - J Hum Lact; doi: 10.1177/0890334412459804}

Information on maternal age, race, smoking status and parity and infant birth weight, gestational age and admission to hospital was obtained from the Perinatal Statistics Collection. Clinical Information Services (CIS) at the WCH is responsible for maintaining the Perinatal Statistics Collection. Data is collected regarding the pregnancy and outcome of every live birth and late fetal death occurring at the $\mathrm{WCH}$, including data on every infant admitted to the neonatal intensive care unit (NICU) and special care baby unit (SCBU). The data are collected according to the guidelines of the Pregnancy Outcome Unit of the South Australian Department of Health for the Supplementary Birth Records (SBRs) and in consultation with the senior clinicians at the $\mathrm{WCH}$. The information in the Perinatal Statistics Collection has been previously validated and is reliable when compared with hospital case records. ${ }^{16}$

The estimated length of gestational age at delivery is recorded in the Perinatal Statistics Collection and is based on the last menstrual period and ultrasound examination. Parity was defined as no previous deliveries (nulliparity) or 1 or more previous deliveries. Maternal race or ethnic group was classified as Caucasian, Asian or other. Data on smoking are routinely collected during the first antenatal visit and therefore reflects smoking status during early pregnancy. The smoking variable was categorised into non-smokers and smokers. According to documentation in the case notes, women were classified as being admitted to the breastfeeding unit (BFU) and/or being assessed by a lactation consultant.

All data analysis was undertaken on de-identified data. Descriptive statistics were used to report frequencies, means, medians and percentage. Univariate categorical analyses using the chi-square test or Fisher's exact test, where appropriate, were conducted. The length of time between childbirth and the first domperidone dispensing was identified and 


\section{Author's Post-Print Version; Copyright - J Hum Lact; doi: 10.1177/0890334412459804}

presented as median number of days between childbirth to first domperidone dispensing. A quartile regression was performed to assess the length of time from birth to first domperidone dispensing from January 2000 to July 2010.

The number of repeat domperidone dispensings was calculated to investigate any change in domperidone dispensing over the 10 -year period. When calculating the numbers of domperidone treatment courses a mother received, a standard rule of ' 6 days between dispensing dates' was used to identify the next course of domperidone treatment.

Agreement between the directions recorded on the original prescriptions filed in the case notes were compared to directions recorded in the dispensing data before and after the introduction of the standardised domperidone prescription chart.

Statistical analyses were performed using SPSS 19 (Statistical Package for the Social Sciences) and STATA 11 (Stata, College Station, TX). Statistical significance was defined as a 2sided $P$ value of 0.05 . This project was approved by the Human Research Ethics Committees of The University of South Australia and the Women's and Children's Health Network in South Australia (RAH 465A).

\section{Results}

\section{Database Audit}

A total of 1,605 (3.6\%) women who delivered at the WCH between January 2000 and July 2010 were dispensed domperidone. Characteristics of women who were dispensed domperidone are shown in Table 1. The mean age of women dispensed domperidone was 30.5 years $( \pm 6.2)$. The majority of women were Caucasian $(79 \%)$, primiparous $(60 \%)$, nonsmokers (78\%) and had normal vaginal delivery (56\%). 


\section{Author's Post-Print Version; Copyright - J Hum Lact; doi: 10.1177/0890334412459804}

The number of women dispensed domperidone increased steadily over the 10-year period from less than $1 \%$ in 2000 to approximately 5\% in 2010 (Figure 1). Over the 10-year period there was no significant difference in the number of days between childbirth and first domperidone dispensing, with the majority of women (94\%) dispensed domperidone at least 4 or more days following childbirth. A further sub-analysis demonstrated, however, that multiparous women were more likely to be dispensed domperidone within 3 days following childbirth compared to primiparous women ( $8 \%$ vs. $4 \%$; $\mathrm{P}<0.01)$. The majority of women included in the audit were dispensed a single course of domperidone (81\%), with the proportion of women receiving multiple courses of domperidone remaining consistent over time. Mothers of preterm infants were more likely to receive a repeat domperidone dispensing than mothers of term infants ( $35 \%$ vs. $10 \%$; $P<0.01)$

\section{Case Note Audit}

Out of the 261 pairs of mother and infant case notes randomly selected, 215 pairs of case notes (82.4\%) were reviewed. Forty-six pairs of case notes were unavailable from medical records. When maternal, or infant, or both case notes were unavailable, the pair of case notes was excluded.

For approximately one third (31\%) of women dispensed domperidone it was documented that they were admitted to the breastfeeding unit, with a further $9 \%$ documented as being assessed by a lactation consultant. Mothers of preterm infants were more likely to be admitted to the breastfeeding unit and/or be assessed by a lactation consultant than mothers of term infants ( $63 \%$ vs. $19 \%$; $P<0.01)$. In addition, primiparous women were more likely to be admitted to the breastfeeding unit and/or be assessed by a lactation consultant than multiparous women (50\% vs. $25.8 \% ; \mathrm{P}<0.01)$. 


\section{Author's Post-Print Version; Copyright - J Hum Lact; doi: 10.1177/0890334412459804}

It was documented that a small percentage (5\%) of women directly requested a prescription for domperidone with a sub-analysis demonstrating that multiparous women were more likely to directly request domperidone than primiparous women (9.0\% vs. $2.4 \%$; $\mathrm{P}=0.03)$.

Furthermore, prior to the introduction of the standardised domperidone dosetapering schedule in 2005 , there was $93 \%$ agreement between the directions on the original prescription and those on the dispensed medication. After 2005, there was $75 \%$ agreement between the directions on the original prescription and those on the dispensed medication. This decrease in the level of agreement was statistically significant $(P=0.03)$. The most commonly dispensed direction over thde 10 year period was the standardised domperidone dose-tapering schedule (96.4\%).

\section{Discussion}

From January 2000 to July 2010 there was a steady increase in the number of women dispensed domperidone at the $\mathrm{WCH}$, from less than $1 \%$ of all pregnancies in 2000 to approximately 5\% in 2010. The infrequent use of domperidone between 2000 and 2002 may have been due to the preferred use of metoclopramide as the first line treatment for managing insufficient breast milk supply during that time. The publication of a randomised controlled trial highlighting the effectiveness of domperidone in increasing milk supply in $2001,{ }^{2}$ could explain the steady increase in domperidone use from 2002 onwards. In response to the apparent increase in demand, a patient information sheet titled 'Medications for increasing breast milk supply' was introduced at WCH in March 2003. The introduction of this patient information sheet may have led to increased dispensing due to increased patient and prescriber awareness of the use of domperidone for this indication. 


\section{Author's Post-Print Version; Copyright - J Hum Lact; doi: 10.1177/0890334412459804}

Only half of the infants identified in the current study were preterm and/or admitted to the NICU or SCBU. This is despite the fact that the majority of previous studies evaluating the use of domperidone as a galactagogue comprised of mothers of infants who were preterm and/or admitted to NICU's, ${ }^{2,5,12}$ )

The standardised domperidone dose-tapering schedule, introduced in 2005 , was the most commonly used direction at the $\mathrm{WCH}$, with the majority of women prescribed domperidone for a total of 12 days. Previously published literature provides inconsistent evidence regarding the most appropriate dose, duration, population and time to initiate domperidone treatment, making it difficult to generate clear evidence-based guidelines for the use of domperidone as a galactagogue. However, anecdotal evidence and clinical experiences recommended taking domperidone for at least six weeks in order to observe its effectiveness. ${ }^{17}$

Given evidence that not all women respond to domperidone and that that the use of domperidone should be discontinued if it does not produce the desired response, ${ }^{18}$ it could be questioned whether a 12 day course is sufficient for evaluating whether domperidone would be effective in increasing milk production and supply. The fact that more than $30 \%$ of mothers who delivered preterm infants received more than one treatment course of domperidone adds further evidence to suggest a longer initial treatment course could be required to ensure maximal effectiveness.

Anecdotally, a reduction in the average length of a postnatal hospital stay at the $\mathrm{WCH}$ could be expected to result in an increasing number of women dispensed domperidone earlier following delivery. However, the number of days between delivery and first domperidone dispensing did not change over the 10 -year period. Given 2 to 3 days is 


\section{Author's Post-Print Version; Copyright - J Hum Lact; doi: 10.1177/0890334412459804}

required for lactation to become well established, ${ }^{19}$ the use of domperidone within the first 3 days following delivery could be considered inappropriate. It is positive that few women (5.5\%) received a domperidone dispensing within the first 3 days following delivery.

However, as it was documented that only a third of these women were admitted to the breastfeeding unit and/or saw a lactation consultant, it raises concerns as to whether women are being dispensed domperidone prior to the trial of appropriate non-pharmacological interventions to increase milk production and supply.

It is interesting to note that multiparous women were more likely to receive a domperidone dispensing within 3 days following delivery compared to primiparous women. It could be speculated that multiparous women, with lactation difficulties in a previous pregnancy, may have received domperidone treatment earlier as they may expect to reexperience similar breastfeeding difficulties and therefore request domperidone earlier. Conversely, primiparous women will have to first attempt breastfeeding themselves prior to realising they have difficulties breastfeeding ${ }^{20}$ and may also be naive to the existence of domperidone and its use as a galactagogue. This is also in accordance with the current finding that multiparous women were more likely to directly request domperidone than primiprous women.

Following the introduction of the standardised dose-tapering schedule, there appeared to be an increased level of disagreement between original prescription directions and those recorded in the dispensing database. In the majority of cases (primarily post 2005), the original directions were changed to reflect those of the standard dose-tapering schedule commonly used at the WCH. The standard dosing schedule may not always be appropriate, as it does not enable the prescriber to individually tailor treatment (i.e. the individual may 


\section{Author's Post-Print Version; Copyright - J Hum Lact; doi: 10.1177/0890334412459804}

require a higher dose, or longer duration of treatment). Importantly, it appears that no information was documented in the maternal or infant case note regarding the change in dispensing directions, or if the original prescriber was consulted regarding the change. It is possible that the original prescriber was contacted and that changes were only made on the copy sent to the pharmacy department and not documented in the patient's case notes. Therefore, it is of concern that women may become confused if the original directions on the prescription are subsequently changed when dispensed by the pharmacist.

It is clear that domperidone should not be used as a substitute for, or as an alternative to, effective non-pharmacological breastfeeding support and encouragement. It is imperative for women who have breastfeeding difficulties to be assessed by a lactation consultant and/or be admitted to BFU to ensure modifiable risk factors of poor breastfeeding are addressed prior to the prescription of any galactagogue. It is of concern that only $40 \%$ of mother's dispensed domperidone were admitted to the BFU and/or received specialist advice from a lactation consultant. However, it is possible that the number of women who received advice from a lactation consultant was underestimated, due to poor documentation in case notes and/or the possibility that some women were assessed by a lactation consultant outside of the WCH.

There are several limitations in the current study. Firstly, this study did not include a control group of women who did not receive a dispensing for domperidone. It was therefore not possible to assess and identify risk factors for lactation failure. Secondly is the reliance on WCH pharmacy dispensing data to determine domperidone use. The total use of domperidone among the cohort of women in the current study is likely to be underestimated, as some women may have been prescribed and dispensed domperidone 


\section{Author's Post-Print Version; Copyright - J Hum Lact; doi: 10.1177/0890334412459804}

outside of the WCH. Without prospectively following a cohort of women after delivery, there is no way to identify how domperidone is being prescribed in the community setting. Dispensing directions were not always available. For example, inpatient supplies of domperidone are not dispensed with directions. In addition, dispensing directions were not available after September 2009 due to a change in the dispensing database program. Thirdly, not all case notes identified in the original random sampling procedure were available to examine, and the quality of data collected was dependant on the quality of documentation in case notes. Lastly, not all of the original prescriptions could be found in the case notes.

This audit of domperidone use as a galactagogue highlights a number of key areas for future research. There is clear need to ensure all women have access to appropriate nonpharmacological support and advice regarding breastfeeding. Furthermore, there is a need to identify the most effective time to initiate domperidone treatment after delivery, recognising that this may be different for primiparous and multiparous women. In addition, further evaluation is required on the most appropriate dose and duration of treatment and whether dose tapering is required and how this should be undertaken. It would also be useful to investigate why some women do and do not respond to treatment with domperidone, which may allow further medication tailoring.

\section{Conclusion}

In conclusion, the retrospective audit at $\mathrm{WCH}$ indicates an overall increase in the percentage of mothers being dispensed domperidone in the last 10 years. With a lack of clinical evidence to guide use, current practice appears to be based on anecdotal evidence, highlighting the need for well-designed randomised controlled trials to support evidencebased practice on the use of domperidone as a galactagogue. 


\section{Funding and Conflict of Interest}

The authors have indicated that they have no financial relationships or conflicts of interest relevant to this article to disclose.

\section{References}

1. Gartner LM, Eidelman Al. Breastfeeding and the Use of Human Milk. Pediatrics. $2005 ; 115(2): 496-506$.

2. da Silva OP, Knoppert DC, Angelini MM, Forret PA. Effect of domperidone on milk production in mothers of premature newborns: a randomized, double-blind, placebocontrolled trial. CMAJ. 2001;164(1):17-21.

3. Chandran L, Gelfer P. Breastfeeding: the essential principles. Pediatr Rev. $2006 ; 27(11): 409-17$.

4. Spinner MR. Maternal-infant bonding. Can Fam Physician. 1978;24:1151-3.

5. Wan EW, Davey K, Page-Sharp M, Hartmann PE, Simmer K, llett KF. Dose-effect study of domperidone as a galactagogue in preterm mothers with insufficient milk supply, and its transfer into milk. Br J Clin Pharmacol. 2008;66(2):283-9.

6. Davis MK. Breastfeeding and chronic disease in childhood and adolescence. Ped Clin North Am. 2001;48(1):125-41.

7. Australian Health Ministers' Conference. The Australian National Breastfeeding Strategy 2010-2015. Canberra: Australian Government Department of Health and Ageing; 2009. 


\section{Author's Post-Print Version; Copyright - J Hum Lact; doi: 10.1177/0890334412459804}

8. Amir LH. Breastfeeding: Managing 'supply' difficulties. Aust Fam Physician. 2006;35(9):686-9.

9. Zuppa AA, Sindico P, Orchi C, Carducci C, Cardiello V, Catenazzi P, et al. Safety and efficacy of galactogogues: substances that induce, maintain and increase breast milk production. J Pharm Pharm Sci. 2010;13(2):162-74.

10. Jones W, Breward S. Use of domperidone to enhance lactation: what is the evidence? Community Pract. 2011;84(6):35-7.

11. Henderson A. Domperidone. Discovering new choices for lactating mothers. AWHONN Lifelines. 2003;7(1):54-60.

12. Campbell-Yeo ML, Allen AC, Joseph K, Ledwidge JM, Caddell K, Allen VM, et al. Effect of Domperidone on the Composition of Preterm Human Breast Milk. Pediatrics. 2010;125(1):e107-e14.

13. Petraglia F, De Leo V, Sardelli S, Pieroni ML, D'Antona N, Genazzani AR. Domperidone in defective and insufficient lactation. Eur J Obstet Gynecol Reprod Biol. 1985;19(5):281-7.

14. Betzold CM. Galactagogues. J Midwifery Womens Health. 2004;49(2):151-4.

15. Gabay MP. Galactogogues: Medications That Induce Lactation. J Hum Lact. 2002 August 1, 2002;18(3):274-9.

16. McLean A, Scott J, Keane R, Sage L, Chan A. Validation of the 1994 South Australian perinatal data collection form. Adelaide, South Australia: Pregnancy Outcome Unit, Epidemiology Branch, Dept. of Human Services; 2001.

17. Newman J, Kernerman E. Domperidone, Getting Started. www.breastfeedinginc.ca/content.php?pagename=doc-DGS. Accessed July 17, 2011. 
18. Brodribb W. Galactagogues. Australian Breastfeeding Association Web Site. www.breastfeeding.asn.au/bfinfo/galact.html. Accessed August 23, 2011.

19. Marshall AM, Nommsen-Rivers LA, Hernandez LL, Dewey KG, Chantry CJ, Gregerson $\mathrm{KA}$, et al. Serotonin transport and metabolism in the mammary gland modulates secretory activation and involution. J Clin Endocrinol Metab. 2010;95(2):837-46.

20. Bystrova K, Widstrom A, Matthiesen AS, Ransjo-Arvidson A, Welles-Nystrom B, Vorontsov I, et al. Early lactation performance in primiparous and multiparous women in relation to different maternity home practices. A randomised trial in St. Petersburg. Int Breastfeed J. 2007;2(9). 
Table 1. Characteristics of Mothers who received a domperidone dispensing

\begin{tabular}{|c|c|}
\hline Mean maternal age $( \pm S D)$, years & $30.5 \pm 6.2$ \\
\hline \multicolumn{2}{|l|}{ Race, n (\%): } \\
\hline Caucasian & $1262(79)$ \\
\hline Aboriginal & $46(3)$ \\
\hline Asian & $214(13)$ \\
\hline Other & $82(5)$ \\
\hline \multicolumn{2}{|l|}{ Parity, n (\%): } \\
\hline Primiparous & $956(60)$ \\
\hline Multiparous & $642(40)$ \\
\hline \multicolumn{2}{|l|}{ Smoking status, $\mathrm{n}(\%)$} \\
\hline Smoker & $263(16)$ \\
\hline Non smoker & $1258(78)$ \\
\hline \multicolumn{2}{|l|}{ Method of delivery, $\mathrm{n}(\%)$} \\
\hline Normal vaginal delivery & $899(56)$ \\
\hline Caesarean delivery & $706(44)$ \\
\hline $\begin{array}{l}\text { Number of days between delivery and domperidone } \\
\text { dispensing, } \mathrm{n}(\%)\end{array}$ & \\
\hline $0-3$ & $89(6)$ \\
\hline$\geq 4$ & $1,516(95)$ \\
\hline Single Course of Domperidone dispensed, $\mathrm{n}(\%)$ & $1,300(81)$ \\
\hline Mother admitted to breastfeeding unit, $\mathrm{n}(\%) \dagger$ & $67(31)$ \\
\hline
\end{tabular}


Author's Post-Print Version; Copyright - J Hum Lact; doi: 10.1177/0890334412459804

\begin{tabular}{|l|l|}
\hline Mother assessed by lactation consultant, but not & $19(9)$ \\
\hline Domperidone directly requested by mother, $\mathrm{n}(\%)^{\dagger}$ & $11(5)$ \\
\hline
\end{tabular}

† Percentages are calculated using the total number of women whose case notes were reviewed $(n=215)$ as the denominator 
Table 2. Characteristics of Infants of Mothers who received a dispensing for domperidone

\begin{tabular}{|l|l|}
\hline $\begin{array}{l}\text { Gestational age, } \mathrm{n}(\%): \\
<37 \text { weeks }\end{array}$ & $660(41)$ \\
$\geq 37$ weeks & $945(59)$ \\
$<2500 \mathrm{~g}$ & $583(36)$ \\
$\geq 2500 \mathrm{~g}$ & $1022(64)$ \\
\hline Birth weight, $\mathrm{n}(\%):$ & $833(52)$ \\
\hline Ydmitted to Neonatal Ward (e.g. NICU), $\mathrm{n}(\%):$ & $772(48)$ \\
\hline No & \\
\hline
\end{tabular}




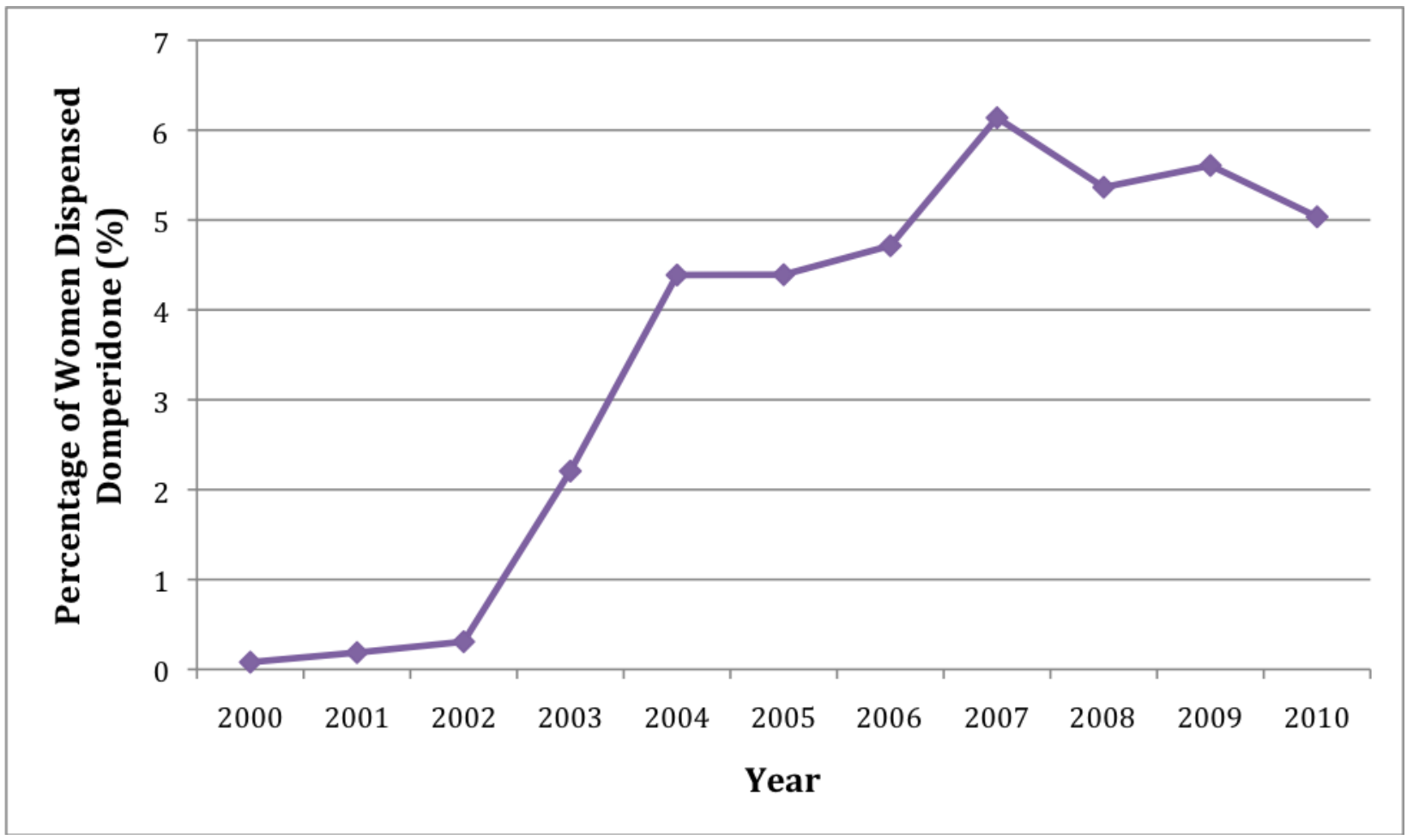

Figure 1. Percentage of women dispensed domperidone according to total number of pregnancies between January 2000 to July 2010 
Author's Post-Print Version; Copyright - J Hum Lact; doi: 10.1177/0890334412459804 